\title{
Analytical Particular Solutions of Multiquadrics Associated with Polyharmonic Operators
}

\author{
Chia-Cheng Tsai \\ Department of Marine Environmental Engineering, National Kaohsiung Marine University, Kaohsiung 811, Taiwan \\ Correspondence should be addressed to Chia-Cheng Tsai; tsaichiacheng@gmail.com
}

Received 9 January 2013; Accepted 29 March 2013

Academic Editor: Kue-Hong Chen

Copyright (c) 2013 Chia-Cheng Tsai. This is an open access article distributed under the Creative Commons Attribution License, which permits unrestricted use, distribution, and reproduction in any medium, provided the original work is properly cited.

\begin{abstract}
We derive two- and three-dimensional analytical particular solutions of multiquadrics (MQ) associated with the polyharmonic operators, named as the polyharmonic multiquadrics (PMQs). The methods of undetermined coefficients are constructed by observing the first few orders of the PMQs which are obtained by the symbolic software, Mathematica. By expanding the PMQs into the Laurent series, the unknown coefficients of the PMQs can be determined. The homogeneous parts of the PMQs are suitably arranged so that the PMQs are hierarchically unique and infinitely differentiable. Mathematica codes are provided for obtaining the PMQs of arbitrary orders. The derived PMQs are validated by numerical solutions for Poisson's equation. Numerical results indicate that the solutions obtained by the PMQs are more accurate than those by the MQ.
\end{abstract}

\section{Introduction}

A radial basis function (RBF) is a real-valued function whose value depends only on the distance from a certain prescribed center. Traditionally, sums of RBFs are used for approximating given functions or reconstructing scattered data. Among the various RBFs, the Gaussian function, Hardy's multiquadrics (MQ) [1], Duchon's augmented polyharmonic spline (APS) [2], and Wendland's compactly supported RBF (CSRBF) [3] are the most popular ones. In the early 1990s, Kansa [4,5] made the first attempt to use the MQ for approximating the solutions of partial differential equations (PDEs). The Kansa method is meshless, simple and has been used for a wide range of PDEs.

Alternatively, Nardini and Brebbia developed the dual reciprocity method (DRM) for approximating the particular of the considered PDE by the RBFs, and then solved the complementary problem by the boundary element method (BEM) [6]. In addition to the BEM, there are also other boundary-type numerical methods such as the method of fundamental solutions (MFS) [7-11] and the Trefftz methods $[12,13]$.

In an early development of DRM, the ad hoc function, $1+r$, was exclusively used. In order to improve the accuracy of the computation, researchers applied the RBF theory to the
DRM [14, 15]. For example, Golberg [9, 16], Chen [17], and Karur and Ramachandran [15] demonstrated the superiority of the APS over the ad hoc trial function. Then, Golberg et al. [18] further improved the accuracy of the approximated particular solution by utilizing the exponential convergence rate of the MQ. A comprehensive review on the DRM development in this period was given by Golberg and Chen [19].

When the MQ is adopted in a DRM procedure, the applicability depends on the availability of the analytical particular solution associated with the partial differential operator of a given problem. Golberg et al. [18] derived the analytical particular solutions for the Laplace equation. Samaan and Rashed $[20,21]$ found the analytical particular solutions for the two-dimensional problems of elasticity and elastodynamics, which could be converted to a biharmonic equation using the Galerkin-Papkovich vector [22]. Basically, the applicability of MQ is limited to the harmonic and biharmonic operators as shown in Yao's thesis [23]. However, Yao's method cannot be easily generalized since the method she applied to cancel the singularity is not straightforward for polyharmonic operators.

In the appendix of a recent study [24], the present author has found analytical particular solutions of the MQ associated with the fourth- and third-ordered polyharmonic operators of two and three dimensions, respectively. In this study, 
the theory for deriving these polyharmonic multiquadrics (PMQs) is presented. In other words, we derive the analytical particular solutions of multiquadrics (MQ) associated with the two- and three-dimensional polyharmonic operators. The methods of undetermined coefficients are constructed by observing the first few orders of the PMQs which are obtained by straight integrations via the symbolic software, Mathematica. Furthermore, the Laurent expansions are used to find the unknown coefficients and to make the PMQs infinitely differentiable. Numerical experiments are carried out to validate the derived PMQs and to demonstrate the superior accuracy of the PMQs over the MQ for solving Poisson's equation.

This paper is organized as follows: the problem is mathematically modeled in Section 2. Then, the two- and three-dimensional PMQs are derived in Sections 3 and 4, respectively. Some numerical experiments are carried out to validate the PMQ in Section 5 and the conclusions are drawn in Section 6.

\section{Definition of the Problem}

In this study, we derive the two- and three-dimensional polyharmonic multiquadrics (PMQs) $\psi_{L}^{(2 D)}$ and $\psi_{L}^{(3 D)}$ of order $L$, which are governed by

$$
\Delta_{r}^{(*)} \psi_{L}^{(*)}=\psi_{L-1}^{(*)}
$$

with

$$
\begin{gathered}
\psi_{0}^{(*)}=\sqrt{c^{2}+r^{2}}, \\
\Delta_{r}^{(2 D)}=\frac{1}{r} \frac{d}{d r}\left(r \frac{d}{d r}\right), \\
\Delta_{r}^{(3 D)}=\frac{1}{r^{2}} \frac{d}{d r}\left(r^{2} \frac{d}{d r}\right) .
\end{gathered}
$$

In (1), (2), and the following, (*) stands for $(2 D)$ or $(3 D)$. If we make the following change of variables

$$
\begin{gathered}
r=c R, \\
\psi_{L}^{(*)}=c^{(2 L+1)} \Psi_{L}^{(*)},
\end{gathered}
$$

equations (1) and (2), respectively, become

$$
\begin{aligned}
& \Delta_{R}^{(*)} \Psi_{L}^{(*)}=\Psi_{L-1}^{(*)}, \\
& \Psi_{0}^{(*)}=\sqrt{1+R^{2}} .
\end{aligned}
$$

Here, $\Delta_{R}^{(*)}$ is defined as (3) while replacing $r$ by $R$. Equations (7) and (6) can be deduced to

$$
\left(\Delta_{R}^{(*)}\right)^{L} \Psi_{L}^{(*)}=\sqrt{1+R^{2}} .
$$

It is clear that the solutions of (6)-(7) are also the solutions of (8). However, the converse is not always true since the homogeneous solutions of (8) can be arbitrarily chosen. In the next two sections, a specific choice will be made to ensure the solutions of (8) are also the solutions of (6)-(7). Then, the PMQs governed by (1)-(2) can be obtained by using (5).

\section{Two-Dimensional PMQ}

It is clear that the solution of (8) can be found by straight integrations. For the two-dimensional case, it can be expressed as follows:

$$
\Psi_{L}^{(2 D)}=\left(\mathbf{I} R^{-1} \mathbf{I} R\right)^{L} \sqrt{1+R^{2}},
$$

where the integral operator $\mathbf{I}$ is defined as $\int d R$. When observing the first few orders of the PMQs obtained by the symbolic software, Mathematica [25], we can find that

$$
\begin{aligned}
& \Psi_{L}^{(2 D)} \\
& =\sum_{j=0}^{L} A_{L, j}^{(2 D)} R^{2 j} \sqrt{1+R^{2}}+\sum_{j=0}^{L-1} B_{L, j}^{(2 D)} R^{2 j} \ln \left(\frac{1+\sqrt{1+R^{2}}}{2}\right) \\
& \quad+\sum_{j=0}^{L-1} C_{L, j}^{(2 D)} R^{2 j}+\sum_{j=0}^{L-1} D_{L, j}^{(2 D)} R^{2 j} \ln R,
\end{aligned}
$$

where the first two series form a particular solution with $2 L+1$ undetermined coefficients $A_{L, j}^{(2 D)}$ and $B_{L, j}^{(2 D)}$ and the last two series give the homogeneous solutions with $2 L$ integration constants $C_{L, j}^{(2 D)}$ and $D_{L, j}^{(2 D)}$. To make $\Psi_{L}^{(2 D)}$ infinitely differentiable, we obviously need

$$
D_{L, j}^{(2 D)}=0,
$$

and then (10) becomes

$$
\begin{aligned}
& \Psi_{L}^{(2 D)} \\
& =\sum_{j=0}^{L} A_{L, j}^{(2 D)} R^{2 j} \sqrt{1+R^{2}}+\sum_{j=0}^{L-1} B_{L, j}^{(2 D)} R^{2 j} \ln \left(\frac{1+\sqrt{1+R^{2}}}{2}\right) \\
& \quad+\sum_{j=0}^{L-1} C_{L, j}^{(2 D)} R^{2 j} .
\end{aligned}
$$

In (12), the coefficients $A_{L, j}^{(2 D)}$ and $B_{L, j}^{(2 D)}$ should be determined such that the PMQ $\Psi_{L}^{(2 D)}$ in (12) satisfies (8) and the arbitrary coefficients $C_{L, j}^{(2 D)}$ should be chosen to ensure the solution $\Psi_{L}^{(2 D)}$ satisfies the hierarchical relation (6) and (7). Therefore, we expand (12) in the Maclaurin series as

$$
\begin{aligned}
\Psi_{L}^{(2 D)}= & \sum_{i=0}^{\infty} \sum_{j=0}^{\min \{L, i\}} \frac{(-1)^{i-j+1}(2 i-2 j-3) ! !}{(i-j) ! 2^{i-j}} A_{L, j}^{(2 D)} R^{2 i} \\
& +\sum_{i=1}^{\infty} \sum_{j=0}^{\min \{L-1, i-1\}} \frac{(-1)^{i-j+1}(2 i-2 j-1) ! !}{(i-j) ! 2^{i-j+1}(i-j)} B_{L, j}^{(2 D)} R^{2 i} \\
& +\sum_{i=0}^{L-1} C_{L, i}^{(2 D)} R^{2 i},
\end{aligned}
$$


where $(\cdot) ! !$ is the double factorial defined as

$$
n ! ! \begin{cases}n \cdot(n-2) \cdots 5 \cdot 3 \cdot 1, & n \text { is an odd positive integer } \\ n \cdot(n-2) \cdots 6 \cdot 4 \cdot 2, & n \text { is an even positive integer } \\ 1, & n=0,-1 \\ \frac{(-1)^{(n+1) / 2}}{(-n-2) ! !}, & n \leq-3 \text { is an odd } \\ \text { negative integer. }\end{cases}
$$

The detailed derivation is given in Appendix A. In order to ensure the solution $\Psi_{L}^{(2 D)}$ satisfies the hierarchical relation (6) and (7), we can make the following selection:

$$
\begin{aligned}
C_{L, i}^{(2 D)}= & \sum_{j=0}^{i} \frac{(-1)^{i-j}(2 i-2 j-3) ! ! A_{L, j}^{(2 D)}}{(i-j) ! 2^{i-j}} \\
& +\sum_{j=0}^{i-1} \frac{(-1)^{i-j}(2 i-2 j-1) ! ! B_{L, j}^{(2 D)}}{(i-j) ! 2^{i-j+1}(i-j)} \quad \text { for } i \leq L-1 .
\end{aligned}
$$

Then, (13) becomes

$$
\begin{aligned}
\Psi_{L}^{(2 D)}= & \sum_{i=L}^{\infty} \sum_{j=0}^{L} \frac{(-1)^{i-j+1}(2 i-2 j-3) ! !}{(i-j) ! 2^{i-j}} A_{L, j}^{(2 D)} R^{2 i} \\
& +\sum_{i=L}^{\infty} \sum_{j=0}^{L-1} \frac{(-1)^{i-j+1}(2 i-2 j-1) ! !}{(i-j) ! 2^{i-j+1}(i-j)} B_{L, j}^{(2 D)} R^{2 i} .
\end{aligned}
$$

The PMQ $\Psi_{L}^{(2 D)}$, given in (16) or equivalently in (12) with $C_{L, j}^{(2 D)}$ defined in (15), is the unique infinitely differentiable PMQ with its first $L$ even coefficients of the Maclaurin series being zero if the undetermined coefficients $A_{L, j}^{(2 D)}$ and $B_{L, j}^{(2 D)}$ can be found by (8). The proposed condition can be alternatively stated as

$$
\left(\Delta_{R}^{(2 D)}\right)^{j} \Psi_{L}^{(2 D)}(R \longrightarrow 0)=0 \text { for } j=0,1, \ldots, L-1,
$$

where the following relation has to be considered:

$$
\left(\Delta_{R}^{(2 D)}\right)^{j} R^{2 i}= \begin{cases}4^{j} \frac{i !^{2}}{(i-L) !^{2}} R^{2 i-2 j} & \text { for } i \geq j \\ 0 & \text { for } i<j\end{cases}
$$

Applying $\Delta_{R}^{(2 D)}$ to the PMQ $\Psi_{L}^{(2 D)}$ in (16) and using (18) indicate that $\Psi_{L-1}^{(2 D)}$ also satisfies (17) and of the same form in (16). Therefore, it can be understood that the prescribed PMQ also satisfies the hierarchical relation (6) and (7).
In order to determine $A_{L, j}^{(2 D)}$ and $B_{L, j}^{(2 D)},(16)$ is substituted into (8) which results in

$$
\begin{aligned}
\sum_{i=L}^{\infty} \sum_{j=0}^{L} \frac{(-1)^{i-j+1}(2 i-2 j-3) ! !\left[i !^{2}\right]}{(i-j) ! 2^{i-j-2 L}\left[(i-L) !^{2}\right]} A_{L, j}^{(2 D)} R^{2 i-2 L} \\
\quad+\sum_{i=L}^{\infty} \sum_{j=0}^{L-1} \frac{(-1)^{i-j+1}(2 i-2 j-1) ! !\left[i !^{2}\right]}{(i-j) ! 2^{i-j-2 L+1}(i-j)\left[(i-L) !^{2}\right]} B_{L, j}^{(2 D)} R^{2 i-2 L} \\
=\sum_{i=0}^{\infty} \frac{(-1)^{i-1}(2 i-3) ! !}{i ! 2^{i}} R^{2 i},
\end{aligned}
$$

where (18) and the following Maclaurin series are used:

$$
\sqrt{1+R^{2}}=\sum_{i=0}^{\infty}\left(\begin{array}{c}
\frac{1}{2} \\
i
\end{array}\right) R^{2 i}=\sum_{i=0}^{\infty} \frac{(-1)^{i-1}(2 i-3) ! !}{i ! 2^{i}} R^{2 i},
$$

with $(:)$ being the usual combination formula.

Substituting $i$ by $i+L$ in the left-hand side of (19) and comparing the coefficients of $R^{2 i}$, we have

$$
\begin{aligned}
& \sum_{j=0}^{L} \frac{(-1)^{i+L-j+1}(2 i+2 L-2 j-3) ! !\left[(i+L) !^{2}\right]}{(i+L-j) ! 2^{i-L-j}\left[i !^{2}\right]} A_{L, j}^{(2 D)} \\
& \quad+\sum_{j=0}^{L-1} \frac{(-1)^{i+L-j+1}(2 i+2 L-2 j-1) ! !\left[(i+L) !^{2}\right]}{(i+L-j) ! 2^{i-L-j+1}(i+L-j)\left[i !^{2}\right]} B_{L, j}^{(2 D)} \\
& =\frac{(-1)^{i-1}(2 i-3) ! !}{i ! 2^{i}}
\end{aligned}
$$

or equivalently

$$
\begin{gathered}
\sum_{j=0}^{L}(-1)^{L-j} 2^{L+j} \underbrace{\frac{(i+L) !}{i !}}_{L \text { th orders }} \frac{(i+L) !}{(i+L-j) !} \\
\times \underbrace{\frac{(2 i+2 L-2 j-3) ! !}{(2 i-3) ! !} A_{L, j}^{(2 D)}}_{j \text { th orders }} \\
+\sum_{j=0}^{L-1}(-1)^{L-j} 2^{L+j-1} \frac{(i+L) !}{i !(i+L-j)} \underbrace{\frac{(i+L d e r s}{(i+L) !}}_{(L-1) \text { th orders }} \underbrace{(i+L) !}_{j \text { th orders }} \\
\times \underbrace{\frac{(2 i+2 L-2 j-1) ! !}{(2 i+1)}}_{(2 i-3) \text { th orders }} B_{L, j}^{(2 D)}=1 .
\end{gathered}
$$

The identity (22) should be satisfied for every nonnegative integer $i$ to ensure the PMQ $\Psi_{L}^{(2 D)}$ is the solution of (8). Since the left-hand side of $(22)$ is a $2 L$-ordered polynomial of the variable $i$, it can be used to uniquely determine the $2 L+1$ unknown coefficients $A_{L, j}^{(2 D)}$ and $B_{L, j}^{(2 D)}$ if the system is not 


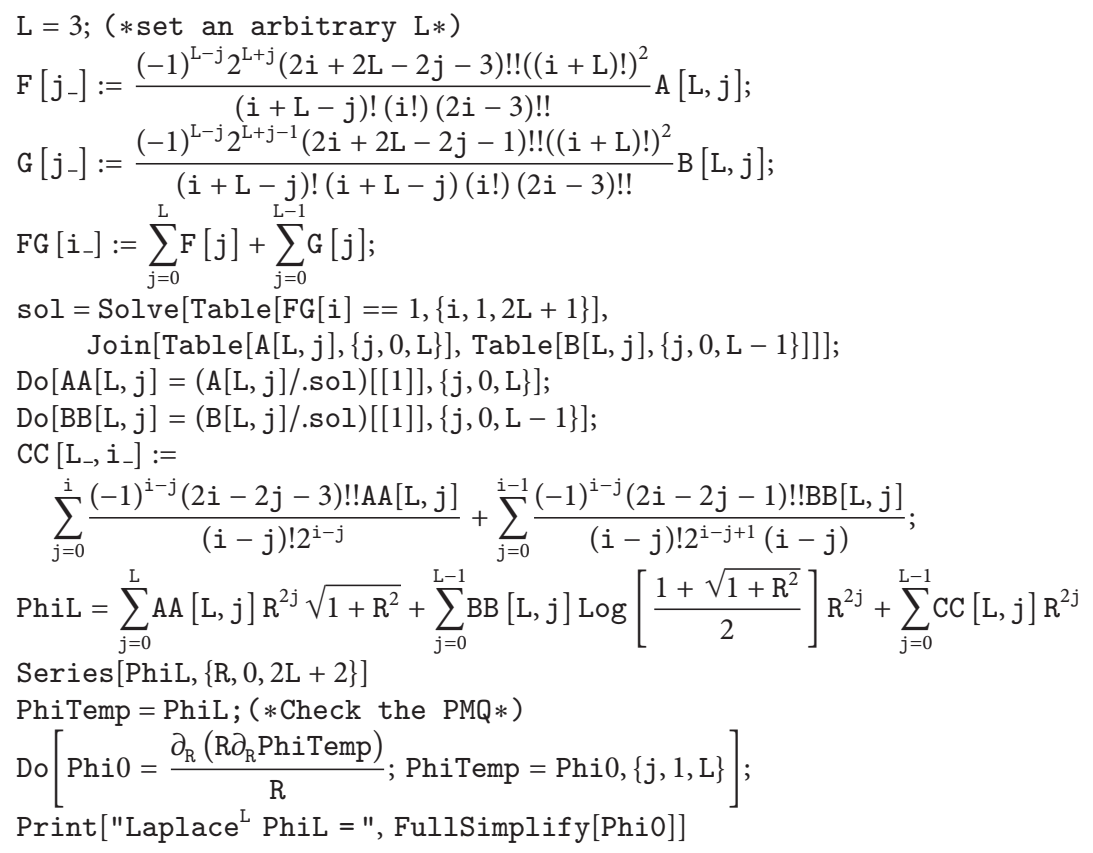

Algorithm 1: Mathematica codes for obtaining 2D-PMQs.

singular. After $A_{L, j}^{(2 D)}$ and $B_{L, j}^{(2 D)}$ are obtained, (15) can be used to have $C_{L, j}^{(2 D)}$. Therefore, the two-dimensional PMQ $\Psi_{L}^{(2 D)}$ can be computed simply by (12). For the cases when $R$ is very small, one can alternatively use (16).

In practice, one can simply set $i$ equal to $1,2, \ldots, 2 L+1$ in (22) which results in $2 L+1$ linear equations for solving the $2 L+1$ unknown coefficients $A_{L, j}^{(2 D)}$ and $B_{L, j}^{(2 D)}$ of the PMQ of order L. Mathematica codes for obtaining the 2D-PMQ are given in Algorithm 1. Alternatively, if a specific low order of 2D-PMQ is considered, one can use the explicit coefficients given in Tables 1, 2, and 3 and the results are plotted in Figure 1. Basically, the PMQs of higher orders are more flat in the near field and grow faster in the far field, as shown in the figure.

\section{Three-Dimensional PMQ}

Similarly, the three-dimensional PMQs can be obtained by the method of undetermined coefficients which begins with

$$
\begin{aligned}
\Psi_{L}^{(3 D)}= & \sum_{j=0}^{L} A_{L, j}^{(3 D)} R^{2 j} \sqrt{1+R^{2}}+\sum_{j=0}^{L-1} B_{L, j}^{(3 D)} R^{2 j} \frac{\sinh ^{-1} R}{R} \\
& +\sum_{j=0}^{L-1} C_{L, j}^{(3 D)} R^{2 j}+\sum_{j=0}^{L-1} D_{L, j}^{(3 D)} R^{2 j-1} .
\end{aligned}
$$

Here, the first two series form a particular solution with undetermined coefficients $A_{L, j}^{(3 D)}$ and $B_{L, j}^{(3 D)}$ and the last two series give the homogeneous solutions with $2 L$ integration constants $C_{L, j}^{(3 D)}$ and $D_{L, j}^{(3 D)}$. Here, it should be noted that the three-dimensional PMQ involving the inverse hyperbolic sine function has been recently derived in the author's previous study [24] and will be further extended in this study. To determine $A_{L, j}^{(3 D)}, B_{L, j}^{(3 D)}, C_{L, j}^{(3 D)}$, and $D_{L, j}^{(3 D)}$, we first expand (23) in terms of the Laurent series as

$$
\begin{aligned}
\Psi_{K, L}^{(3 D)}= & \sum_{i=0}^{\infty} \sum_{j=0}^{\min \{L, i\}} \frac{(-1)^{i-j-1}(2 i-2 j-3) ! !}{(i-j) ! 2^{i-j}} A_{L, j}^{(3 D)} R^{2 i} \\
& +\sum_{i=0}^{\infty} \sum_{j=0}^{\min \{L-1, i\}} \frac{(-1)^{i-j}(2 i-2 j-1) ! !}{(i-j) ! 2^{i-j}(2 i-2 j+1)} B_{L, j}^{(3 D)} R^{2 i} \\
& +\sum_{i=0}^{L-1} C_{L, i}^{(3 D)} R^{2 i}+\sum_{i=0}^{L-1} D_{L, i}^{(3 D)} R^{2 i-1} .
\end{aligned}
$$

The detailed derivation is given in Appendix B. In order to make $\Psi_{L}^{(3 D)}$ infinitely differentiable, it is required to cancel the singular term $R^{-1}$ and all the odd coefficients in (24) [26]. In other words, we need

$$
D_{L, i}^{(3 D)}=0
$$

Similar to (17), we additionally consider the following relation:

$$
\left(\Delta_{R}^{(3 D)}\right)^{j} \Psi_{L}^{(3 D)}(R \longrightarrow 0)=0 \quad \text { for } j=0,1, \ldots, L-1
$$




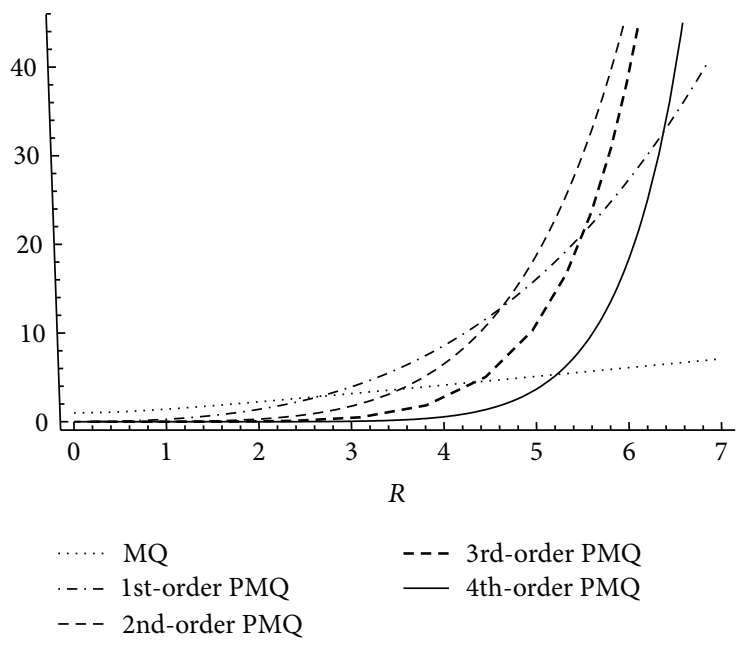

(a)

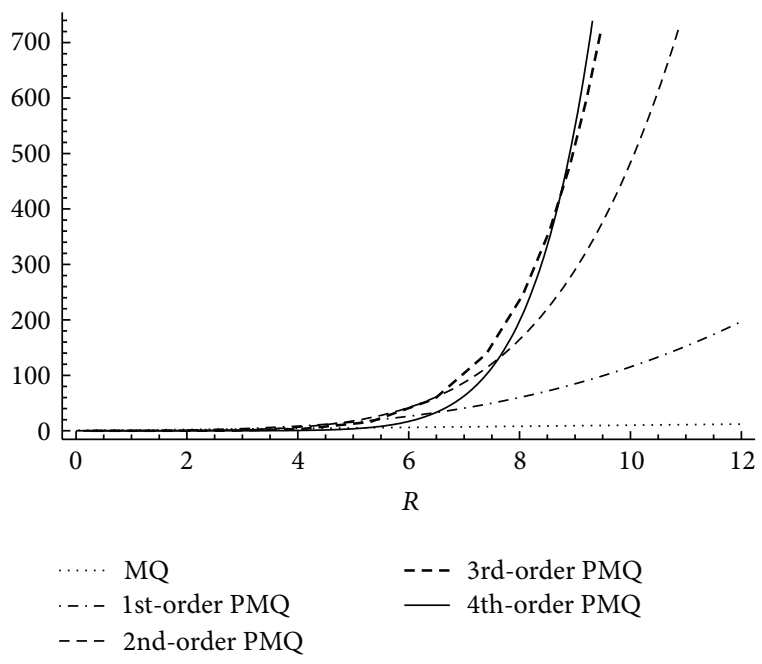

(b)

FIgURe 1: Plots of the 2D-PMQs in (a) near field and (b) far field.

TABLE 1: $A_{L, j}^{(2 D)}$

\begin{tabular}{|c|c|c|c|c|c|c|}
\hline & $j=0$ & $j=1$ & $j=2$ & $j=3$ & $j=4$ & $j=5$ \\
\hline$L=1$ & $\frac{4}{9}$ & $\frac{1}{9}$ & & & & \\
\hline$L=2$ & $-\frac{61}{900}$ & $\frac{4}{75}$ & $\frac{1}{225}$ & & & \\
\hline$L=3$ & $\frac{1019}{352800}$ & $-\frac{153}{15680}$ & $\frac{8}{3675}$ & $\frac{1}{11025}$ & & \\
\hline$L=4$ & $-\frac{3407}{57153600}$ & $\frac{229}{510300}$ & $-\frac{33007}{76204800}$ & $\frac{8}{178605}$ & $\frac{1}{893025}$ & \\
\hline$L=5$ & $\frac{80939}{110649369600}$ & $\frac{9535}{983549952}$ & $\frac{735263}{35407798272}$ & $-\frac{3311969}{354077982720}$ & $\frac{4}{7203735}$ & $\frac{1}{108056025}$ \\
\hline
\end{tabular}

TABLE 2: $B_{L, j}^{(2 D)}$.

\begin{tabular}{lccccc}
\hline & $j=0$ & $j=1$ & $j=2$ & $j=3$ & $j=4$ \\
\hline$L=1$ & $-\frac{1}{3}$ & \\
$L=2$ & $\frac{1}{30}$ & $-\frac{1}{12}$ & \\
$L=3$ & $-\frac{1}{840}$ & $\frac{1}{120}$ & $-\frac{1}{192}$ & \\
$L=4$ & $\frac{1}{45360}$ & $-\frac{1}{3360}$ & $\frac{1}{1920}$ & $-\frac{1}{6912}$ & \\
$L=5$ & $-\frac{1}{3991680}$ & $\frac{1}{181440}$ & $-\frac{1}{53760}$ & $\frac{1}{69120}$ & $-\frac{1}{442368}$ \\
\hline
\end{tabular}

TABle 3: $C_{L, j}^{(2 D)}$.

\begin{tabular}{lccccc}
\hline & $j=0$ & $j=1$ & $j=2$ & $j=3$ & $j=4$ \\
\hline$L=1$ & $-\frac{4}{9}$ & & & \\
$L=2$ & $\frac{61}{900}$ & $-\frac{1}{36}$ & & \\
$L=3$ & $-\frac{1019}{352800}$ & $\frac{31}{3600}$ & $\frac{1}{1152}$ & & \\
$L=4$ & $\frac{3407}{57153600}$ & $-\frac{599}{1411200}$ & $\frac{1}{3600}$ & $\frac{1}{13824}$ & \\
$L=5$ & $\frac{80939}{110649369600}$ & $\frac{2147}{228614400}$ & $-\frac{389}{22579200}$ & $\frac{1}{345600}$ & $\frac{1}{589824}$
\end{tabular}


with

$$
\left(\Delta_{R}^{(3 D)}\right)^{j} R^{2 i}= \begin{cases}\frac{(2 i+1) !}{(2 i-2 j+1) !} R^{2 i-2 j} & \text { for } i \geq j \\ 0 & \text { for } i<j .\end{cases}
$$

Then we use (24) and (26) to have the following relation:

$$
\begin{aligned}
C_{L, i}^{(3 D)}= & \sum_{j=0}^{i} \frac{(-1)^{i-j}(2 i-2 j-3) ! ! A_{L, j}^{(3 D)}}{(i-j) ! 2^{i-j}} \\
& +\sum_{j=0}^{i} \frac{(-1)^{i-j-1}(2 i-2 j-1) ! ! B_{L, j}^{(3 D)}}{(i-j) ! 2^{i-j}(2 i-2 j+1)} \quad \text { for } i \leq L-1 .
\end{aligned}
$$

Substituting (28) into (24) gives

$$
\begin{aligned}
\Psi_{L}^{(3 D)}= & \sum_{i=L}^{\infty} \sum_{j=0}^{L} \frac{(-1)^{i-j-1}(2 i-2 j-3) ! !}{(i-j) ! 2^{i-j}} A_{L, j}^{(3 D)} R^{2 i} \\
& +\sum_{i=L}^{\infty} \sum_{j=0}^{L-1} \frac{(-1)^{i-j}(2 i-2 j-1) ! !}{(i-j) ! 2^{i-j}(2 i-2 j+1)} B_{L, j}^{(3 D)} R^{2 i} .
\end{aligned}
$$

The PMQ $\Psi_{L}^{(3 D)}$, given in (29) or equivalently in (23) with $C_{L, i}^{(3 D)}$ and $D_{L, i}^{(3 D)}$ defined in (28) and (25), is the only infinitely differentiable PMQ satisfying (26) if the undetermined coefficients $A_{L, j}^{(3 D)}$ and $B_{L, j}^{(3 D)}$ can be found by using (8). Then, we consider the following relation.

Equation (27) with $j=1$ together with (29) is sufficient to infer that the prescribed PMQ also satisfies the hierarchical relation (6) and (7) as explained in the two-dimensional case. Substituting (29) into (8) and using (20) and (27) result in

$$
\begin{aligned}
& \sum_{i=L}^{\infty} \sum_{j=0}^{L} \frac{(-1)^{i-j-1}(2 i-2 j-3) ! !(2 i+1) !}{(i-j) ! 2^{i-j}(2 i-2 L+1) !} A_{L, j}^{(3 D)} R^{2 i-2 L} \\
& \quad+\sum_{i=L}^{\infty} \sum_{j=0}^{L-1} \frac{(-1)^{i-j}(2 i-2 j-1) ! !(2 i+1) !}{(i-j) ! 2^{i-j}(2 i-2 j+1)(2 i-2 L+1) !} B_{L, j}^{(3 D)} R^{2 i-2 L} \\
& =\sum_{i=0}^{\infty} \frac{(-1)^{i-1}(2 i-3) ! !}{i ! 2^{i}} R^{2 i} .
\end{aligned}
$$

Substituting $i$ by $i+L$ for the left-hand side of (30) and comparing the coefficients of $R^{2 i}$, we have

$$
\begin{aligned}
& \sum_{j=0}^{L} \frac{(-1)^{i+L-j-1}(2 i+2 L-2 j-3) ! !(2 i+2 L+1) ! A_{L, j}^{(3 D)}}{(i+L-j) ! 2^{i+L-j}(2 i+1) !} \\
& \quad+\sum_{j=0}^{L-1} \frac{(-1)^{i+L-j}(2 i+2 L-2 j-1) ! !(2 i+2 L+1) ! B_{L, j}^{(3 D)}}{(i+L-j) ! 2^{i+L-j}(2 i+2 L-2 j+1)(2 i+1) !} \\
& =\frac{(-1)^{i-1}(2 i-3) ! !}{i ! 2^{i}}
\end{aligned}
$$

or equivalently

$$
\begin{aligned}
\sum_{j=0}^{L}(-1)^{L-j} 2^{j} \underbrace{\frac{(i+L) !}{(i+L-j) !}}_{\frac{(2 i+2 L+1) ! !}{(2 i+1) ! !}} \underbrace{(i+r s}_{j \text { th orders }} \\
\times \\
+\underbrace{\frac{(2 i+2 L-2 j-3) ! !}{(2 i-3) ! !}}_{(L-j) \text { th orders }} A_{L, j}^{(3 D)} \\
+\underbrace{\sum^{L-1}(-1)^{L-j+1} 2^{j} \frac{(2 i+2 L+1) ! !}{(2 i+1) ! !(2 i+2 L-2 j+1)}}_{j=0} \underbrace{\frac{(i+L-j) !}{(i-1) \text { th orders }}}_{j \text { th orders }} \\
\times \frac{(2 i+2 L-2 j-1) ! !}{(2 i-3) ! !} B_{L, j}^{(3 D)}=1 .
\end{aligned}
$$

Similar to the two-dimensional cases, (32) can be used to uniquely determine the $2 L+1$ unknown coefficients $A_{L, j}^{(3 D)}$ and $B_{L, j}^{(3 D)}$ since its left-hand side is a $2 L$-ordered polynomial of the variable $i$. Similarly, Mathematica codes for obtaining the 3D-PMQ are given in Algorithm 2. Also, the first few orders of 3D-PMQ are given in Tables 4, 5, and 6 and plotted in Figure 2. In the figure, we can also observe that the PMQs of higher orders are more flat in the near field and grow faster in the far field.

\section{Validation}

By the standard MFS-DRM, the PMQs derived in this paper have been validated for the cases of fourth and third orders, respectively, in two and three dimensions [24]. The results basically indicate that the solutions of the MQ are more accurate than those of the low-ordered APS. Numerical results for other orders of polyharmonic problems should be similar and thus will not be repeated here.

Alternatively, we apply the PMQs to the standard MFSDRM for solving Poisson's equation

$$
\Delta u=f(\mathbf{x}) ; \quad \mathbf{x} \in \Omega,
$$

where $\Omega \subset \mathbf{R}^{2}$ or $\mathbf{R}^{3}$ is the considered domain, $f$ is the given forcing term, and $\Delta$ denotes the Laplacian operator. For simplicity, we consider the boundary condition

$$
u=g(\mathbf{x}) ; \quad \mathbf{x} \in \Gamma,
$$

where $\Gamma$ is the boundary of $\Omega$ and $g$ is the given boundary data. In the formal MFS-DRM, the solution should be decomposed into two parts

$$
u=u_{p}+u_{h}
$$

where the particular solution $u_{p}$ satisfies

$$
\Delta u_{p}=f(\mathbf{x}) ; \quad \mathbf{x} \in \Omega,
$$




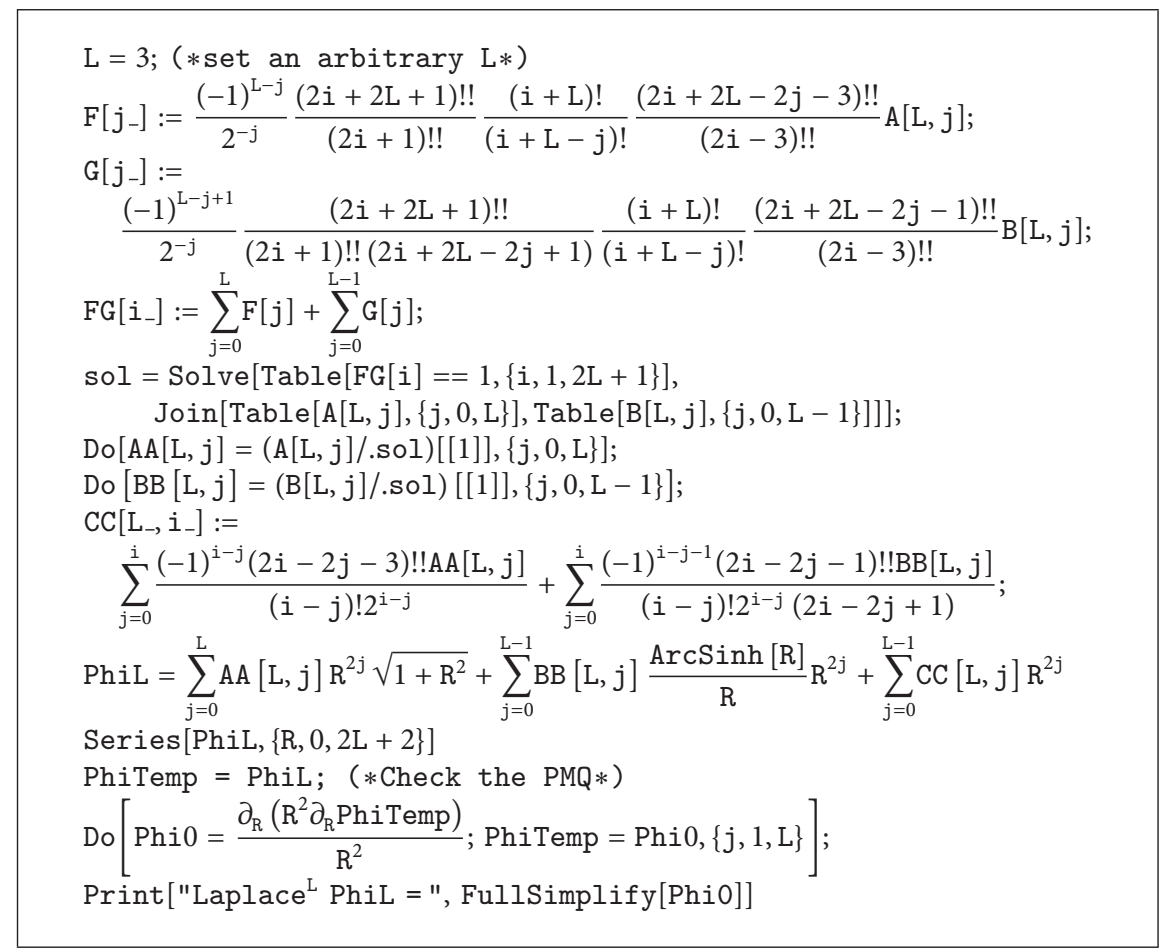

Algorithm 2: Mathematica codes for obtaining 3D-PMQs.

TABLE 4: $A_{L, j}^{(3 D)}$.

\begin{tabular}{|c|c|c|c|c|c|c|}
\hline & $j=0$ & $j=1$ & $j=2$ & $j=3$ & $j=4$ & $j=5$ \\
\hline \multirow{2}{*}{$L=1$} & 5 & 1 & & & & \\
\hline & $\overline{24}$ & $\overline{12}$ & & & & \\
\hline \multirow{2}{*}{$L=2$} & 9 & 7 & 1 & & & \\
\hline & $\overline{160}$ & $\overline{360}$ & $\overline{360}$ & & & \\
\hline \multirow{2}{*}{$L=3$} & 919 & 391 & 3 & 1 & & \\
\hline & $\overline{322560}$ & 53760 & $\overline{4480}$ & $\overline{20160}$ & & \\
\hline \multirow{2}{*}{$L=4$} & 3781 & 5777 & 1457 & 11 & 1 & \\
\hline & $\overline{58060800}$ & $\overline{14515200}$ & $\overline{4838400}$ & $\overline{907200}$ & $\overline{1814400}$ & \\
\hline \multirow{2}{*}{$L=5$} & 122987 & 406247 & 926801 & 55151 & 13 & 1 \\
\hline & $\overline{143061811200}$ & $\overline{42918543360}$ & $\overline{53648179200}$ & $\overline{8941363200}$ & $\overline{95800320}$ & 239500800 \\
\hline
\end{tabular}

TABLE 5: $B_{L, j}^{(3 D)}$.

\begin{tabular}{lccccc}
\hline & $j=0$ & $j=1$ & $j=2$ & $j=3$ & $j=4$ \\
\hline$L=1$ & $\frac{1}{8}$ & & & \\
$L=2$ & $-\frac{1}{96}$ & $\frac{1}{16}$ & & \\
$L=3$ & $\frac{1}{3072}$ & $-\frac{1}{192}$ & $\frac{1}{192}$ & & \\
$L=4$ & $-\frac{1}{184320}$ & $\frac{1}{6144}$ & $-\frac{1}{2304}$ & $\frac{1}{5760}$ & \\
$L=5$ & $\frac{1}{17694720}$ & $-\frac{1}{368640}$ & $\frac{1}{73728}$ & $-\frac{1}{69120}$ & $\frac{1}{322560}$ \\
\hline
\end{tabular}

but not necessarily the boundary condition (34). And the homogeneous solution $u_{h}$ is defined by

$$
\begin{gathered}
\Delta u_{h}=0 ; \quad \mathbf{x} \in \Omega, \\
u_{h}=g(\mathbf{x})-u_{p} ; \quad \mathbf{x} \in \Gamma .
\end{gathered}
$$

The solution of (37) can be formally obtained by the MFS [711] and will not be additionally described.

On the other hand, in order to solve the particular solution, we approximate the forcing term by

$$
f(\mathbf{x}) \approx \sum_{i=1}^{M} \alpha_{i} \psi_{L}^{(*)}\left(r_{i}\right),
$$




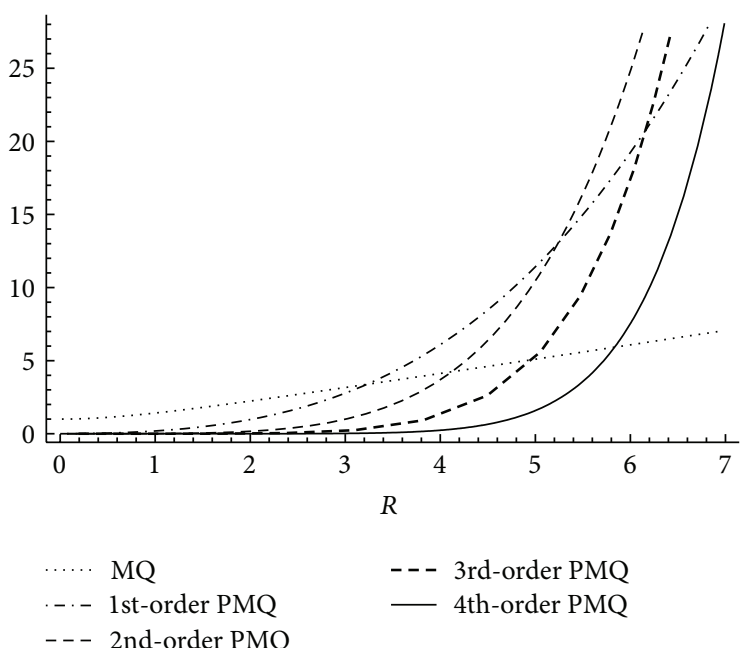

(a)

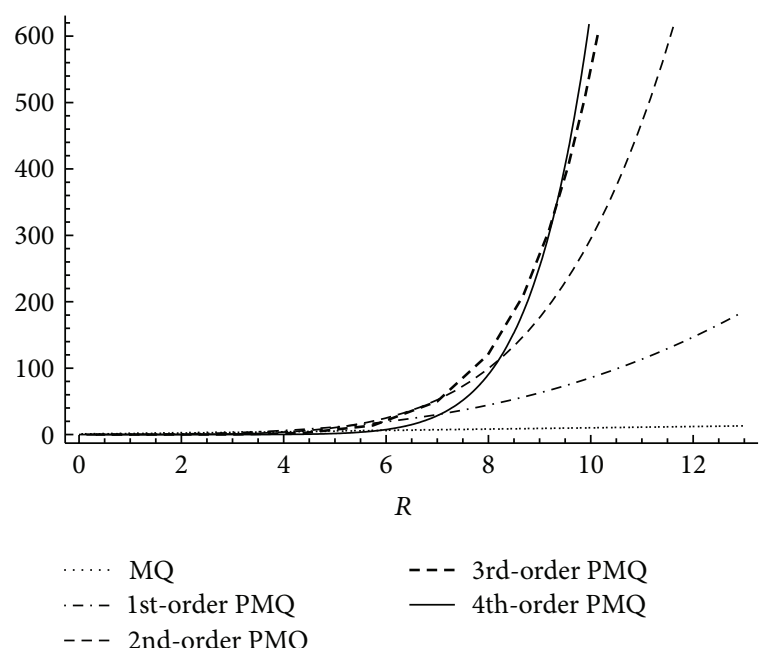

(b)

FIgURe 2: Plots of the 3D-PMQs in (a) near field and (b) far field.

TABLE 6: $C_{L, j}^{(3 D)}$.

\begin{tabular}{lccccc}
\hline & $j=0$ & $j=1$ & $j=2$ & $j=3$ & $j=4$ \\
\hline$L=1$ & $-\frac{1}{3}$ & & & \\
$L=2$ & $\frac{1}{15}$ & $-\frac{1}{18}$ & & \\
$L=3$ & $-\frac{1}{315}$ & $\frac{1}{90}$ & $-\frac{1}{360}$ & \\
$L=4$ & $\frac{1}{14175}$ & $-\frac{1}{1890}$ & $\frac{1}{1800}$ & $-\frac{1}{15120}$ & \\
$L=5$ & $-\frac{1}{1091475}$ & $\frac{1}{85050}$ & $-\frac{1}{37800}$ & $\frac{1}{75600}$ & $-\frac{1}{1088640}$ \\
\hline
\end{tabular}

where $r_{i}=\left\|\mathbf{x}-\mathbf{x}_{i}\right\|$ is the Euclidean distance between the spatial coordinates $\mathbf{x}$ and $M$ prescribed points $\mathbf{x}_{i}$ in $\Omega$ and $\psi_{L}^{(*)}$ is the PMQ of order $L$. In order to solve the unknown coefficients $\alpha_{i}$, (38) should be collocated on the $M$ prescribed points $\mathbf{x}_{i}$ as

$$
f\left(\mathbf{x}_{j}\right)=\sum_{i=1}^{M} \alpha_{i} \psi_{L}^{(*)}\left(r_{i j}\right) \text { for } j=1,2, \ldots, M
$$

where $r_{i j}=\left\|\mathbf{x}_{j}-\mathbf{x}_{i}\right\|$. After the forcing term is approximated in (38), the particular solution can be correspondingly approximated as

$$
u_{p}(\mathbf{x}) \approx \sum_{i=1}^{M} \alpha_{i} \psi_{L+1}^{(*)}\left(r_{i}\right)
$$

For $L=0$, the solution procedure is reduced to the formal MFS-DRM based on the MQ [18].
5.1. Two-Dimensional Case. We consider a two-dimensional problem governed by Poisson's equation (33) with $\Omega=$ $[-(1 / 2), 1 / 2]^{2}$ and

$$
\begin{aligned}
f(\mathbf{x})= & f(x, y)=4 \cos \left(2 x^{2}+y\right) \\
& -\left(1+16 x^{2}\right) \sin \left(2 x^{2}+y\right) .
\end{aligned}
$$

The boundary condition (34) is set according to the exact solution as

$$
u(x, y)=g(x, y)=\sin \left(2 x^{2}+y\right) .
$$

Since the maximum value of the solution in $\Omega$ is equal to $\sin 1 \approx 0.84$, we use the maximum error in this example. And the sources of the MFS are typically located on the boundary of a square with edge length equal to 5 .

We have hierarchically performed our tests for $L=$ $0,1,2, \ldots, 8$. Among the similar results, the maximum errors for $L=0,2,4,6,8$ are addressed in Table 7 . This should have validated the two-dimensional PMQs for $L=0,1,2, \ldots, 9$. For every individual test, a preliminary search is always performed for finding the optimal shape parameter $c$ as demonstrated in Figure 3. It can be observed from Table 7 that the best maximum errors (marked by $*$ ) usually occur at $L=6$ and are about an order of magnitude better than those obtained by the MQ and the case of more collocation points (larger $M$ ) gives better accuracy. If we observe the PMQ coefficients, we will find that eight is the minimum order of the PMQ with some coefficients over the limit of 64bit unsigned integer. To be more detailed, $A_{8,5}^{(2 D)}$ is equal to $1457447323 / 28298491779317760000$ whose denominator is just larger than $2^{64}-1 \approx 1.8 \times 10^{19}$.

5.2. Three-Dimensional Case. Then, we consider a threedimensional problem governed by Poisson's equation (33) with $\Omega=[-(1 / 2), 1 / 2]^{3}$ and

$$
f(\mathbf{x})=f(x, y, z)=\frac{6}{(4+x+y+z)^{3}} .
$$




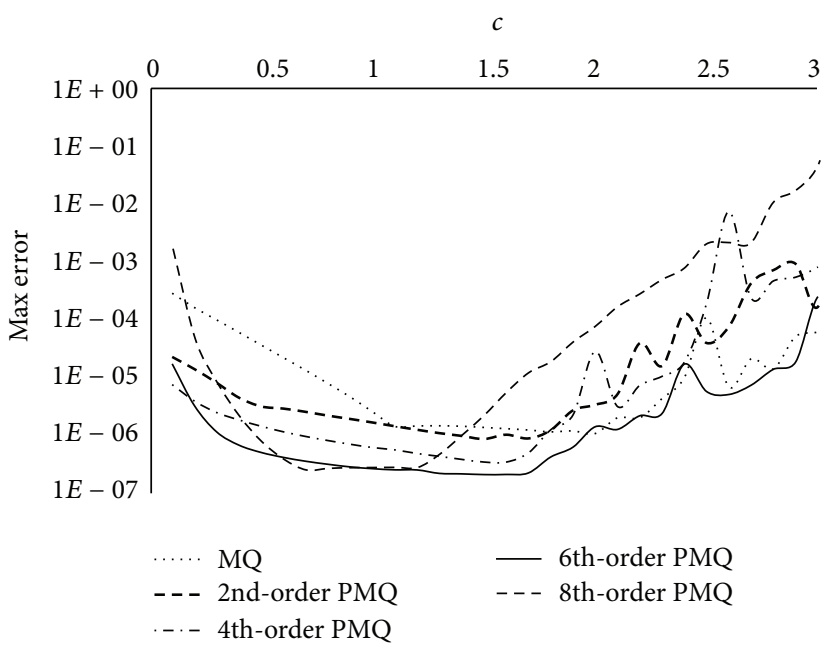

(a)

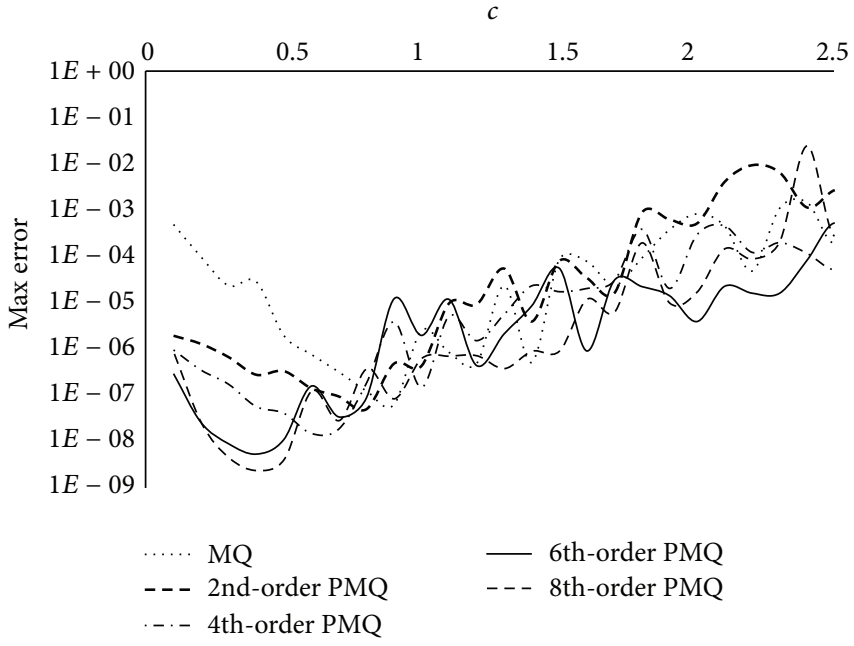

(b)

FIGURE 3: Search for the optimal shape parameter $c$ for the 2D case with (a) $M=9 \times 9$ and (b) $M=13 \times 13$.

TABle 7: Maximum errors of the two-dimensional Poisson's problem.

\begin{tabular}{lccc}
\hline & $M=9 \times 9$ & $M=11 \times 11$ & $M=13 \times 13$ \\
\hline$L=0$ & $1.07 E-06$ & $1.64 E-07$ & $6.38 E-08$ \\
$L=2$ & $8.23 E-07$ & $1.22 E-07$ & $4.99 E-08$ \\
$L=4$ & $3.50 E-07$ & $2.85 E-08$ & $1.47 E-08$ \\
$L=6$ & $2.08 E-07^{*}$ & $1.12 E-08^{*}$ & $5.06 E-09$ \\
$L=8$ & $2.53 E-07$ & $1.43 E-08$ & $2.25 E-09^{*}$ \\
\hline
\end{tabular}

${ }^{*}$ Means the best result.

The boundary condition (34) is set according to the exact solution as

$$
u(x, y, z)=g(x, y, z)=\frac{1}{4+x+y+z}
$$

Since the maximum value of the solution in the computational domain is equal to 0.25 , we also use the maximum error in this example. And the sources of the MFS are typically located on the boundary of a cuboid with edge length equal to 5 .

Similarly, we also hierarchically perform numerical tests for $L=0,1,2, \ldots, 8$ and address the maximum errors for $L=0,2,4,6,8$ in Table 8 . This should also validate the threedimensional PMQs for $L=0,1,2, \ldots, 9$. Figure 4 gives the preliminary search for finding the optimal shape parameter $c$. It can also be observed from Table 8 that the best maximum errors basically occur at $L=6$ and are about an order of magnitude better than those obtained by the MQ. Similarly, we have $A_{8,5}^{(3 D)}$ equal to $977222021 / 25635104317734912000$ whose denominator is larger than $2^{64}-1 \approx 1.8 \times 10^{19}$.
TABle 8: Maximum errors of the three-dimensional Poisson's problem.

\begin{tabular}{lccc}
\hline & $M=5 \times 5 \times 5$ & $M=7 \times 7 \times 7$ & $M=9 \times 9 \times 9$ \\
\hline$L=0$ & $1.23 E-06$ & $9.22 E-08$ & $1.73 E-08$ \\
$L=2$ & $2.03 E-06$ & $6.67 E-08$ & $1.66 E-08$ \\
$L=4$ & $4.61 E-07$ & $1.07 E-08$ & $1.02 E-09$ \\
$L=6$ & $2.63 E-07^{*}$ & $3.68 E-08$ & $6.07 E-10^{*}$ \\
$L=8$ & $1.09 E-02$ & $2.03 E-08^{*}$ & $7.55 E-10$ \\
\hline
\end{tabular}

${ }^{*}$ Means the best result.

It seems that the PMQ of higher order gives more accurate results if there is no numerical ill-conditioning. However for the case of $M=5 \times 5 \times 5$ and $L=8$, the error goes worse as shown in Table 8. This result seems to indicate that the PMQ of higher order also requires more collocation points. In practice, one can safely choose $L=4 \sim 6$ and find some improvement over the MQ.

\section{Conclusion}

The polyharmonic multiquadrics (PMQs) are derived. The methods of undetermined coefficients are constructed and the PMQs are solved by the Laurent expansion. Some additional conditions are introduced to make the derived PMQs hierarchically unique and infinitely differentiable. Numerical experiments were carried out to validate the derived PMQs. Basically, the numerical results obtained by the PMQ showed some improvement over those by the multiquadrics.

The method introduced in this study can be extended to derive the polyharmonic particular solutions of the generalized multiquadrics $\left(r^{2}+c^{2}\right)^{K / 2}$ with $K=-1,1,3, \ldots$ which is currently under investigation. 


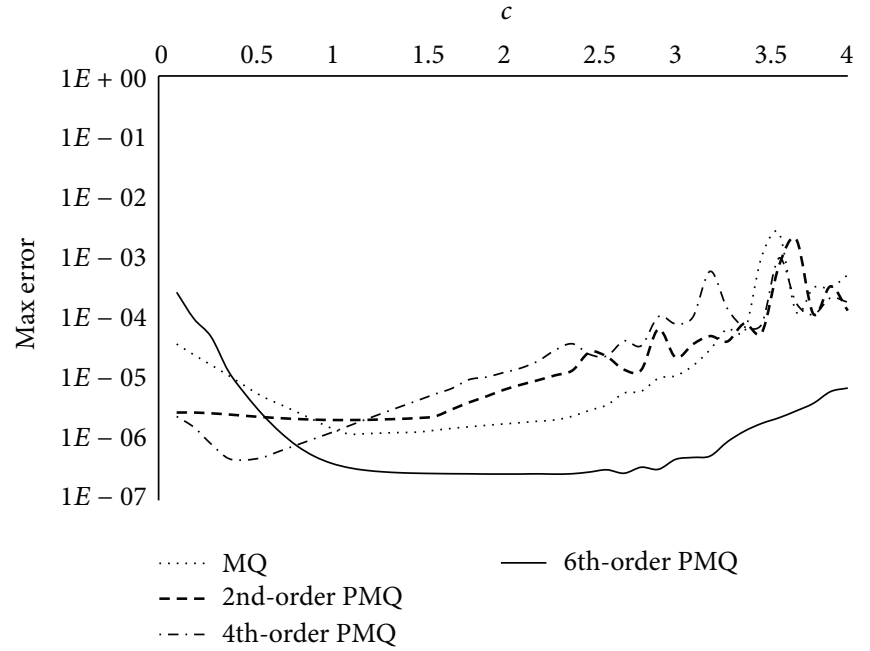

(a)

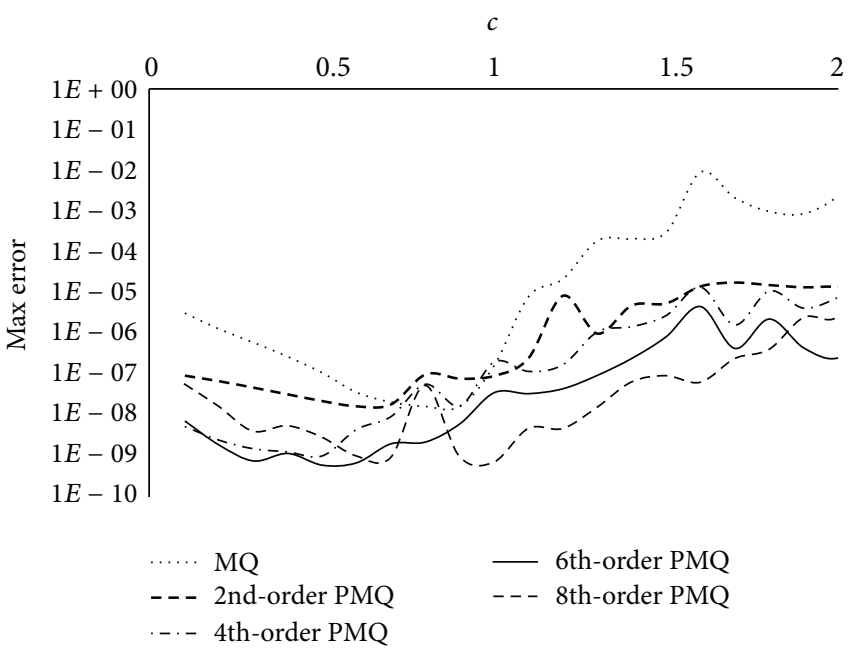

(b)

FIGURE 4: Search for the optimal shape parameter $c$ for the 3D case with (a) $M=5 \times 5 \times 5$ and (b) $M=9 \times 9 \times 9$.

\section{Appendices}

\section{A. Maclaurin Series of (12)}

Since the Maclaurin series of $\left(1+R^{2}\right)^{1 / 2}$ is given in (20), we begin with the Maclaurin series of $\left(1+R^{2}\right)^{-1 / 2}$ as

$$
{\sqrt{1+R^{2}}}^{-1}=\sum_{i=0}^{\infty}\left(\begin{array}{c}
-\frac{1}{2} \\
i
\end{array}\right) R^{2 i}=\sum_{i=0}^{\infty} \frac{(-1)^{i}(2 i-1) ! !}{i ! 2^{i}} R^{2 i} .
$$

Then, we observe the relation

$$
\frac{d}{d R}\left(\ln \frac{1+\sqrt{1+R^{2}}}{2}\right)=\frac{1}{R}\left(1-\frac{1}{\sqrt{1+R^{2}}}\right) .
$$

Equations (A.1) and (A.2) can be combined to have

$$
\ln \left(\frac{1+\sqrt{1+R^{2}}}{2}\right)=\sum_{i=1}^{\infty} \frac{(-1)^{i+1}(2 i-1) ! !}{i ! 2^{i+1} i} R^{2 i} .
$$

Equations (20) and (A.3) are sufficient to find the Maclaurin series of (12).

\section{B. Laurent Series of (23)}

First we observe the relation

$$
\frac{d}{d R}\left(\sinh ^{-1} R\right)=\frac{1}{\sqrt{1+R^{2}}} .
$$

Equations (A.1) and (B.1) can be combined to have

$$
\frac{\sinh ^{-1} R}{R}=\sum_{i=0}^{\infty} \frac{(-1)^{i}(2 i-1) ! !}{i ! 2^{i}(2 i+1)} R^{2 i} .
$$

Equations (20) and (B.2) are sufficient to find the Maclaurin series of (23).

\section{Acknowledgment}

The National Science Council of Taiwan is gratefully acknowledged for providing financial support to carry out the present work under Grant no. NSC 101-2628-E-022-E-MY2.

\section{References}

[1] R. L. Hardy, "Multiquadric equations of topography and other irregular surfaces," Journal of Geopphysical Research, vol. 76, pp. 1905-1915, 1971.

[2] J. Duchon, "Splines minimizing rotation-invariant semi-norms in Sobolev spaces," in Constructive Theory of Functions of Several Variables, vol. 571 of Lecture Notes in Mathematics, pp. 85-100, Springer, Berlin, Germany, 1977.

[3] H. Wendland, "Piecewise polynomial, positive definite and compactly supported radial functions of minimal degree," Advances in Computational Mathematics, vol. 4, no. 4, pp. 389396, 1995.

[4] E. J. Kansa, "Multiquadrics-a scattered data approximation scheme with applications to computational fluid-dynamics. I. Surface approximations and partial derivative estimates," Computers \& Mathematics with Applications, vol. 19, no. 8-9, pp. 127-145, 1990.

[5] E. J. Kansa, "Multiquadrics-a scattered data approximation scheme with applications to computational fluid-dynamics. II. Solutions to parabolic, hyperbolic and elliptic partial differential equations," Computers \& Mathematics with Applications, vol. 19, no. 8-9, pp. 147-161, 1990.

[6] D. Nardini and C. A. Brebbia, "A new approach to free vibration analysis using boundary elements," Applied Mathematical Modelling, vol. 7, no. 3, pp. 157-162, 1983.

[7] A. Bogomolny, "Fundamental solutions method for elliptic boundary value problems," SIAM Journal on Numerical Analysis, vol. 22, no. 4, pp. 644-669, 1985.

[8] G. Fairweather and A. Karageorghis, "The method of fundamental solutions for elliptic boundary value problems," Advances in Computational Mathematics, vol. 9, no. 1-2, pp. 6995, 1998. 
[9] M. A. Golberg, "The method of fundamental solutions for Poisson's equation," Engineering Analysis with Boundary Elements, vol. 16, no. 3, pp. 205-213, 1995.

[10] V. D. Kupradze and M. A. Aleksidze, "The method of functional equations for the approximate solution of certain boundaryvalue problems," USSR Computational Mathematics and Mathematical Physics, vol. 4, no. 4, pp. 82-126, 1964.

[11] R. Mathon and R. L. Johnston, "The approximate solution of elliptic boundary-value problems by fundamental solutions," SIAM Journal on Numerical Analysis, vol. 14, no. 4, pp. 638-650, 1977.

[12] C.-S. Liu, "A highly accurate solver for the mixed-boundary potential problem and singular problem in arbitrary plane domain," Computer Modeling in Engineering \& Sciences, vol. 20, no. 2, pp. 111-122, 2007.

[13] C. S. Liu, "A highly accurate MCTM for direct and inverse problems of biharmonic equation in arbitrary plane domains," Computer Modeling in Engineering \& Sciences, vol. 30, no. 2, pp. 65-75, 2008.

[14] M. A. Golberg and C. S. Chen, "The theory of radial basis functions applied to the bem for inhomogeneous partial differential equations," Boundary Elements Communications, vol. 5, pp. 5761, 1994.

[15] S. R. Karur and P. A. Ramachandran, "Radial basis function approximation in the dual reciprocity method," Mathematical and Computer Modelling, vol. 20, no. 7, pp. 59-70, 1994.

[16] M. A. Golberg, "Numerical evaluation of particular solutions in the BEM-a review," Boundary elements communications, vol. 6 , no. 3, pp. 99-106, 1995.

[17] C. S. Chen, "The method of fundamental solutions for nonlinear thermal explosions," Communications in Numerical Methods in Engineering, vol. 11, no. 8, pp. 675-681, 1995.

[18] M. A. Golberg, C. S. Chen, and S. R. Karur, "Improved multiquadric approximation for partial differential equations," Engineering Analysis with Boundary Elements, vol. 18, no. 1, pp. 9-17, 1996.

[19] M. A. Golberg and C. S. Chen, Eds., The Method of Fundamental Solutions for Potential, Helmholtz and Diffusion Problems, Computational Mechanics Publications, Southampton, UK, 1999.

[20] M. F. Samaan and Y. F. Rashed, "BEM for transient 2D elastodynamics using multiquadric functions," International Journal of Solids and Structures, vol. 44, no. 25-26, pp. 8517-8531, 2007.

[21] M. F. Samaan and Y. F. Rashed, "Free vibration multiquadric boundary elements applied to plane elasticity," Applied Mathematical Modelling, vol. 33, no. 5, pp. 2421-2432, 2009.

[22] A. H. D. Cheng, C. S. Chen, M. A. Golberg, and Y. F. Rashed, "BEM for theomoelasticity and elasticity with body force-a revisit," Engineering Analysis with Boundary Elements, vol. 25, no. 4-5, pp. 377-387, 2001.

[23] G. Yao, The method of approximate particular solutions for solving partial differential equations [Ph.D. thesis], Department of Mathematics, The University of Southern Mississippi, 2010.

[24] C.-C. Tsai, "Automatic particular solutions of arbitrary high-order splines associated with polyharmonic and polyHelmholtz equations," Engineering Analysis with Boundary Elements, vol. 35, no. 7, pp. 925-934, 2011.

[25] S. Wolfram, The Mathematica Book, Cambridge University Press, Cambridge, UK, 1996.

[26] H. Wendland, "On the smoothness of positive definite and radial functions," Journal of Computational and Applied Mathematics, vol. 101, no. 1-2, pp. 177-188, 1999. 


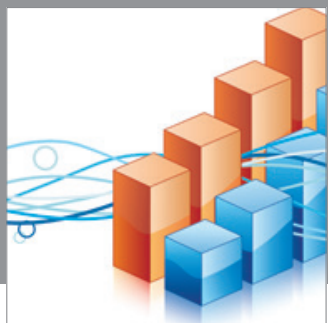

Advances in

Operations Research

mansans

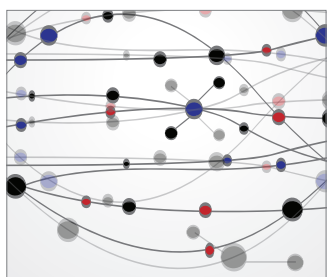

The Scientific World Journal
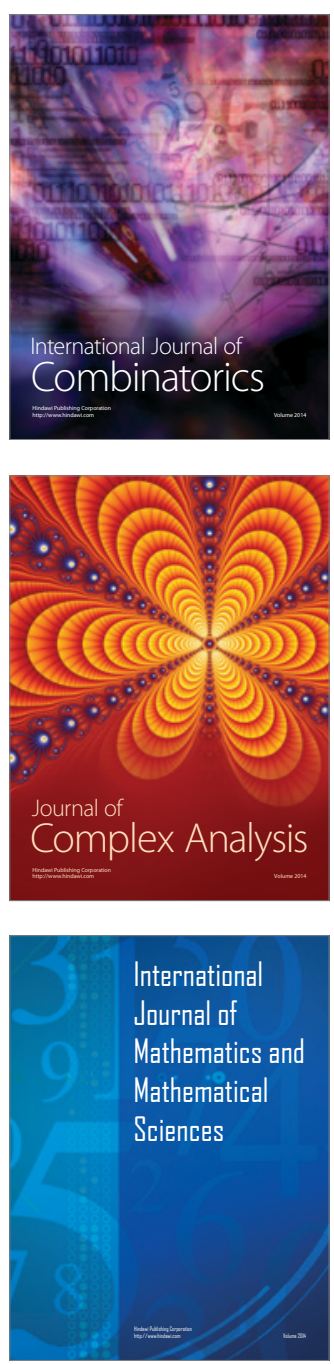
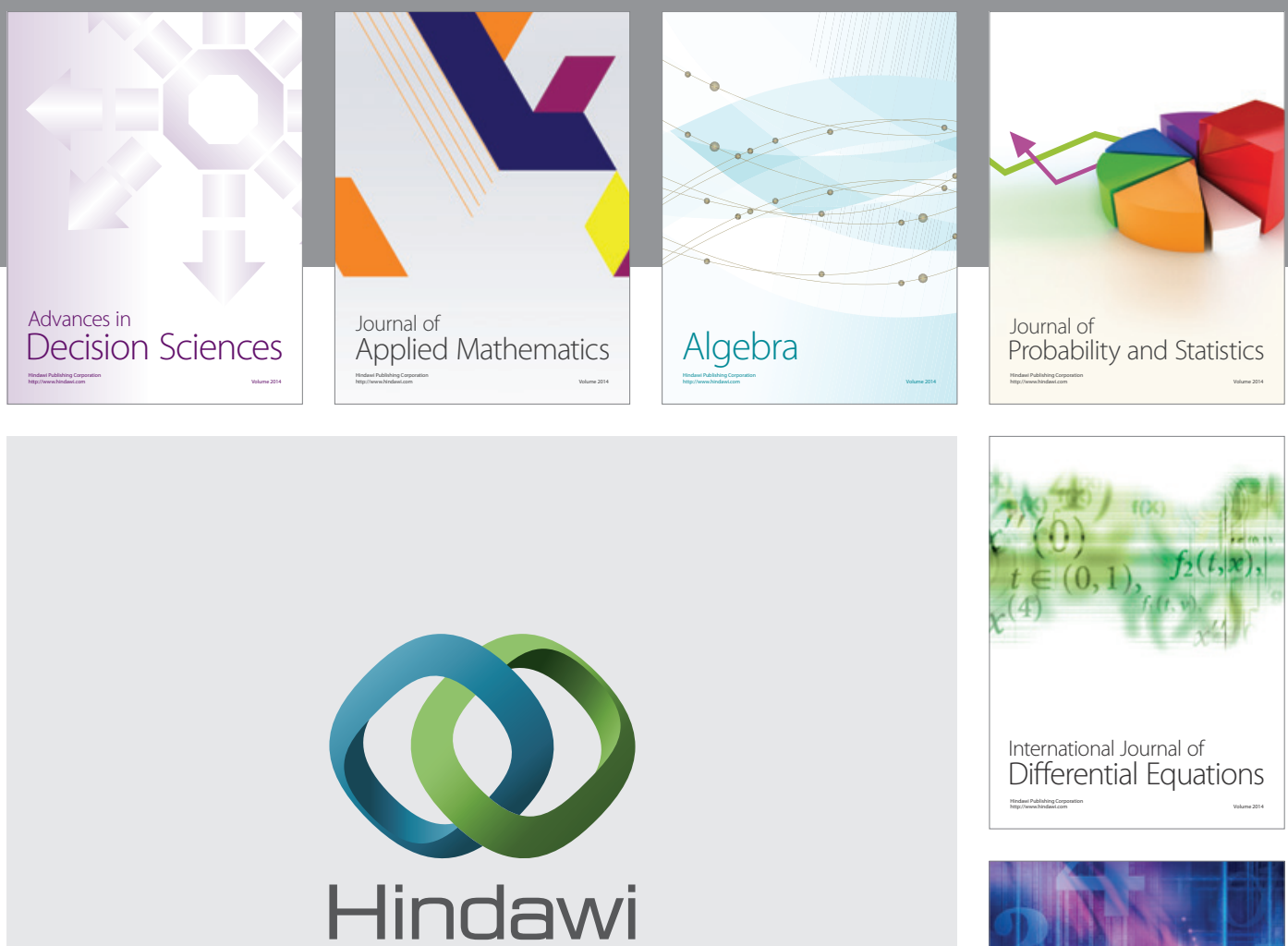

Submit your manuscripts at http://www.hindawi.com
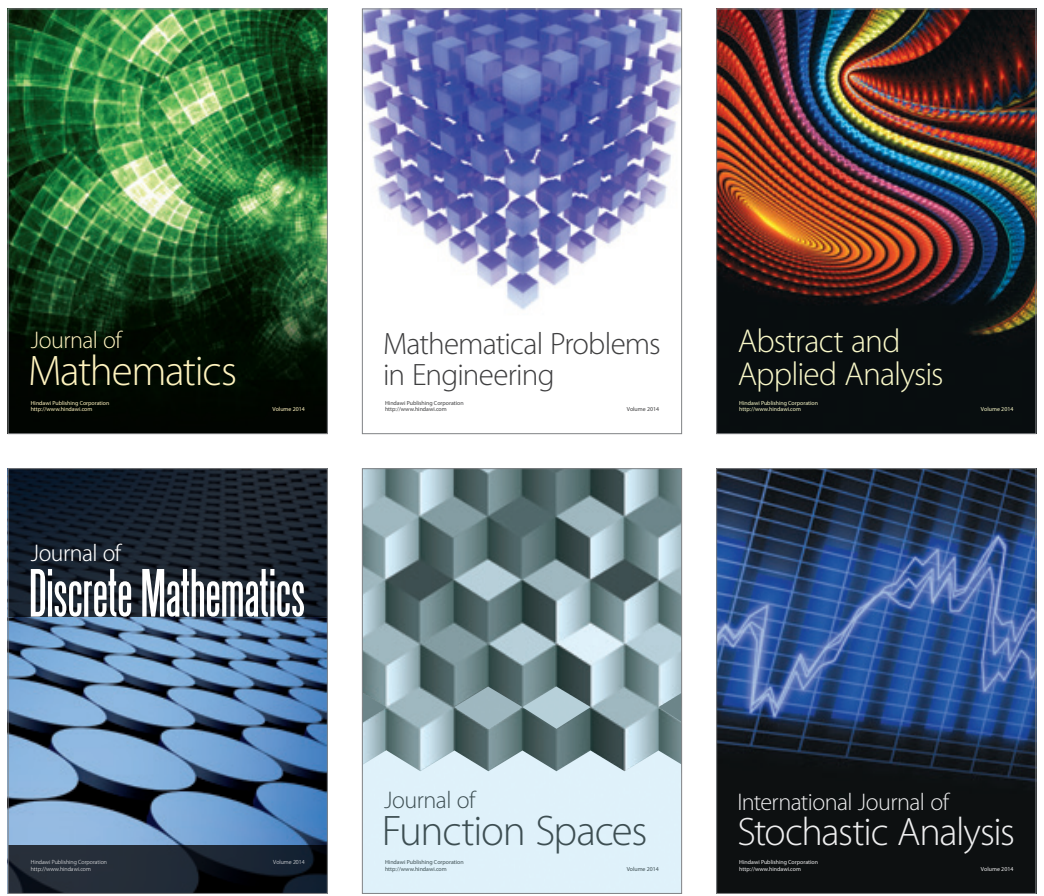

Journal of

Function Spaces

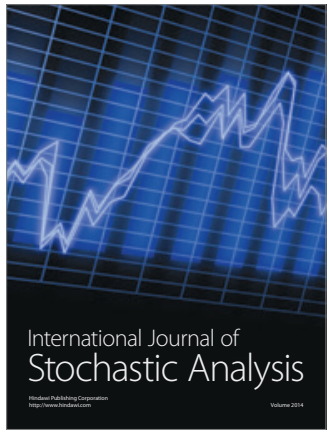

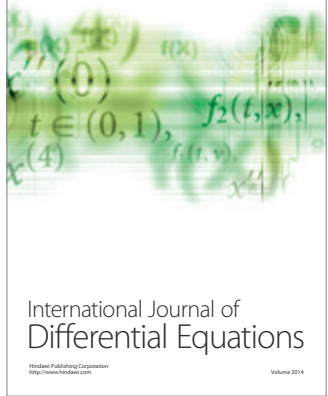
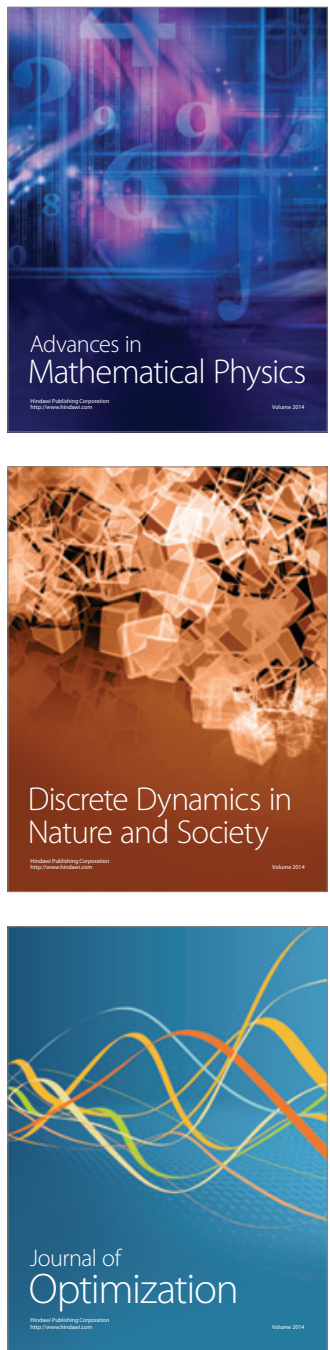